\title{
Familial Recurrent Hydatidiform Moles: A rare case report
}

\author{
Fatma Eskicioglu ${ }^{1}$, Isin Kaya ${ }^{2}$, Esra Bahar Gur ${ }^{3}$, Guluzar Arzu Turan ${ }^{3}$
}

\begin{abstract}
Recurrent hydatidiform moles (RHM) are described as the being of at least two molar pregnancies in the same patient. It is very rare. NLPR7 mutations are found high rate in patients with RHM. KHDC3L is the second responsible gene for RHM. In this paper, we present an interesting case of a familial RHM. Here, we have discussed the genetic counseling to be given to a patient who had been diagnosed as hydatidiform mole in her two previous pregnancies and whose sister had a history of four consecutive molar pregnancies. Because rate of NLPR7 mutation is high in individuals with recurrent molar pregnancy, patients should be recommended to have NLRP7 gene sequence analysis in the first place. If no mutation is detected in this gene, KHDC31 gene sequence analysis should be carried out.
\end{abstract}

Keywords: Recurrent hydatidiform moles, NLPR7, KHDC3L, Mutation

Introduction

Gestational trophoblastic disease is a tumor characterized by proliferation of trophoblast and originating from the placenta. It has a wide spectrum compose of partial (PHM) and complete hydatidiform mole (CHM), invasive mole, choriocarcinoma and placental site trophoblastic tumor. Hydatidiform mole (HM) appears once in every 600 pregnancies in Western countries but at higher rates in the Middle East, Latin America, Africa, and the Far East [1]. HM in next gestation is rare; its probability of occurrence is approximately $1 \%$ in sporadic PHM or CHM [2]. Recurrent hydatidiform moles (RHM) are described as the being of at least two molar pregnancies in the same patient [1]. In this study, we discussed the genetic counseling to be given to a patient who had been diagnosed as HM in her two previous pregnancies and whose sister had a history of four consecutive molar pregnancies.

Case

Twenty seven-year-old patient was admitted with a desire to conceive. Her obstetric history revealed 2 pregnancy losses at $8^{\text {th }}$ week due to missed abortion. The pathologic examination of both curettage materials in those pregnancies were reported as PHM.
No pathologic finding was detected during gynecologic examination. Chromosome analysis of peripheral blood was normal. Family history revealed that patient's sister experienced 4 consecutive molar pregnancies. Patient, who wanted to know whether same condition would recur during her subsequent pregnancies, was recommended to have a NLRP7 gene sequence analysis, and KHDC3L gene sequence, if no mutation is found in this gene.

\section{Discussion}

Familial recurrent hydatidiform mole is an exceedingly rare clinical condition. Recurrent CHM in two Lebanese family members and recurrent PHM in one Lebanese family has been defined by Moglabey et al. [3]. They demonstrated that these molar pregnancies were diploid and genomes were bi-parental. Based on their data and previously reported FRHM cases, they presumed that the women with RHM pregnancy were homozygote for an autosomal recessive mutation. It was claimed that paternal genome did not contribute to development of molar pregnancy in autosomal recessive condition [4]. An extensive genomic screening was performed by the Moglabey et al. on the Lebanese family. Responsibility of FRHM gene

Received: 5 Sept. 2014, Revised 15 Sept. 2014, Accepted 17 Sept. 2014, Available Online 10 Oct. 2014

${ }^{1}$ Merkez effendi State Hospital, Department of Obstetrics and Gynecology, 45050 Manisa, Turkey

${ }^{2}$ Şifa University, School of Medicine, Department of Medical Genetics, 35100 Izmir, Turkey

${ }^{3}$ Şifa University, School of Medicine, Department of Obstetrics and Gynecology, 35100 Izmir, Turkey

*Corresponding Author: Fatma Eskicioglu E-mail: fatmaeskicioglu@gmail.com 
had been demonstrated in order to map of these genes and a maternal gene [3]. After obtaining DNA from blood samples of the family members, a wide genomic screening was carried out with 250 microsatellite marker panel at $10-15 \mathrm{cM}$ interval. It was shown that defective gene was located in a 15,2 cM interval flanked by D19S924 and D19S890 loci in $19 \mathrm{q} 13.3-13.4$

NLRP7 (nucleotide oligomerization domain (NOD)-like receptor, pyrin containing 7) is the first identified gene that causes RHM localized in $19 q 13.4$ region. NLRP7 is seen as a major gene as to screening studies conducted on various groups and populations. Mutation in this gene was detected in $48-80 \%$ of the patients who had at least two molar pregnancies. These mutations contain stop codon, minor deletion or insertions (less than 20 bp), splice mutations, major deletion or insertions and complex rearrangements. In addition to these mutations, two protein truncating mutations, L $823 \mathrm{X}$ stop codon mutation and a deletion from intron 8 through intron 11 and about 17 missense mutation were detected in patients with RHM or sporadic mole as single heterozygote mutation or variant. Pathologic significance of these single mutations and variants is still controversial and further data are needed to reach a conclusion [1]. According to Williams et al [4], homozygote or compound mutations exist in NLRP7 gene in most of the women with familial bi-parental HM, and these results confirm that this gene is a major cause of bi-parental HM.

NLRP7 transcripts were identified in liver, lung, spleen, thymus, placenta, endometrium, hematopoietic cells, testis, and in all oocyte stages and pre-implantation embryos [1. 5]. Knockdown of expression of NLRP7 gene in human embryonic stem cells causes earlier expression of two trophoblastic differentiation marker, GCM1 and INSL4, therefore it is suggested that lack of NLRP7 function alters trophoblastic differentiation. Knockdown of NLRP7 increases the level of HCG. This role of NLRP7 is very important in hydatidiform mole which is characterized by trophoblastic hyper proliferation and increased HCG production [1].

IL-1 $\beta$ secretion decreased in mononuclear cells in NLRP7 mutation or rare variants has been shown by the Messaed et al. [6]. Current data shows some linkages between IL-1 $\beta$ and ovulation and oocyte maturation. As levels of IL-1 $\beta$ increase, related with ovulation rate increases, oocyte and subsequent normal embryonic growth rate decrease. However, this role of IL- $1 \beta$ on oocytes contradicts with data in cells of the patients with NLRP7 mutations. In addition, mice with deficiency of IL$1 \beta$ were found to be fertile, and IL-1 $\beta$ signal deficiency does not affect fertility and embryo viability in mice. As a result, perhaps combination of these roles of NLRP7, some effects on oocytes, effects on embryonic and trophoblastic tissue proliferation and differentiation and other effects on hematopoietic inflammatory cells and down regulation in maternal immune response contribute to HM [1]. According to Nguyen et al. [1]role of NLRP7 in down regulation of maternal inflammation and insufficiency in spontaneous elimination of non-viable pregnancies is a basic aspect that enables differentiation of this disease from other forms of early pregnancy losses.

KHDC3L ( $\mathrm{KH}$ domain containing 3-like) identified in 2011 is the second gene responsible for recurrent HM. KHDC3L is localized on chromosome 6 . It is thought to be the responsible gene in $10-14 \%$ of RHM patients without a mutation in NLRP7. Four mutations have been reported so far. KHDC3L transcripts were identified in all oocyte stages, pre-implantation embryos and many tissues containing hematopoietic cells. Similar to expression profile of NLRP7, expression of KHD3CL is the highest during germinal vesicle phase in oocytes and it drops during pre-implantation process and it becomes undetectable during blastocyst phase [1].

Because the rate of NLPR7 mutation is high in individuals with recurrent molar pregnancy, NLRP7 gene sequence analysis should be recommended for in the first place. If no mutation is detected in this gene, KHDC3L gene sequence analysis should be carried out. For NLRP7 gene, 11 exon PCR products are examined with direct sequence analysis and a comparison is made with NM_001127255.1 sequence. For KHDC3L gene, 3 exon PCR products are examined with direct sequence analysis and comparison is made with NM_001017361 sequence [1].

The purpose of DNA test in these patients is to determine the chance for having a healthy baby or to detect the risks of recurrence of molar pregnancy and malign sequel. A woman who has two defective allele for NLRP7 gene has a rather low potential of delivering a normal alive baby. Nguyen et al. reported only $3(7 \%)$ alive deliveries in 43 cases in their own study groups. No live birth was reported in other

RHM patients who have NLRP7 or KHDC3L mutation. No live birth was reported in few cases that had two defective alleles belonging to KHDC3L gene. NLRP7 and KHDC3L genes are necessary for oocyte. Therefore, in theory, oocyte donation should be suggested in order to improve reproductive outcomes in these patients [1].

In 2011, healthy live birth with oocyte donation in a patient with compound heterozygote mutations of p.E13GfsX7 and p.R693P in NLRP7 gene has been reported by the Fisher RA et al. [7]. This result establishes the major role of NLRP7 gene in pregnancy and oocyte development and provides a hope for normal pregnancy in other women. 
If there are not any mutations in NLRP7 and KHDC3L genes in FRHM cases, currently undetected mutations in these genes or mutations in unidentified genes can be responsible [1].

Genomic screenings involving more cases in large groups will provide more data on genetic origin in HM cases.

Financial Support: This research received no specific grant from any funding agency, commercial or not-for-profit sectors

Conflict of Interest: The authors declared that they had no conflicts of interest.

\section{References}

1. Nguyen NM, Slim R. Genetics and Epigenetics of Recurrent Hydatidiform Moles: Basic Science and Genetic Counselling. Current obstetrics and gynecology reports. 2014;3:55-64

2. Sebire NJ, Fisher RA, Foskett M, Rees H, Seckl MJ, Newlands ES. Risk of recurrent hydatidiform mole and subsequent pregnancy outcome following complete or partial hydatidiform molar pregnancy. BJOG : an international journal of obstetrics and gynaecology. 2003;110(1):22-6

3. Moglabey YB, Kircheisen R, Seoud M, El Mogharbel N, Van den Veyver I, Slim R. Genetic mapping of a maternal locus responsible for familial hydatidiform moles. Human molecular genetics. 1999;8(4):667-71.

4. Williams D, Hodgetts V, Gupta J. Recurrent hydatidiform moles. European journal of obstetrics, gynecology, and reproductive biology. 2010;150(1):3-7.

5. Slim R, Wallace EP. NLRP7 and the Genetics of Hydatidiform Moles: Recent Advances and New Challenges. Frontiers in immunology. 2013;4:242.

6. Messaed C, Akoury E, Djuric U, Zeng J, Saleh M, Gilbert $\mathrm{L}$, et al. NLRP7, a nucleotide oligomerization domain-like receptor protein, is required for normal cytokine secretion and co-localizes with Golgi and the microtubuleorganizing center. The Journal of biological chemistry. 2011;286(50):43313-23

7. Fisher RA, Lavery SA, Carby A, Abu-Hayyeh S, Swingler R, Sebire NJ, et al. What a difference an egg makes. Lancet. 2011;378(9807):1974.

Copyright (C) 2014 The Author(s); This is an open-access article distributed under the terms of the Creative Commons Attribution License (http://creativecommons.org/licenses/by/4.0), which permits unrestricted use, distribution, and reproduction in any medium, provided the original work is properly cited. 